\title{
Accounting for flexibility in power system planning with renewables
}

\author{
Andreas Belderbos, Erik Delarue*
}

University of Leuven (KU Leuven) Energy Institute, TME branch (Applied Mechanics and Energy Conversion), Celestijnenlaan 300A box 2421 / B-3001 Leuven / Belgium

E-mail addresses: andreas.belderbos@kuleuven.be ; erik.delarue@mech.kuleuven.be

* Corresponding author:

Erik Delarue

University of Leuven (KU Leuven) Energy Institute, TME branch (Applied Mechanics and Energy Conversion), Celestijnenlaan 300A box 2421

B-3001 Leuven

Belgium

Tel.: +3216 322511; fax: +3216322985.

erik.delarue@mech.kuleuven.be 


\begin{abstract}
Due to the increasing deployment of intermittent renewables, the residual load profile, as seen by the dispatchable generation units, becomes lower and more volatile. This paper introduces a new system planning model on a power plant resolution, taking into account technical operational constraints. The objective of this model is to determine the optimal set of generation units, able to serve a given demand. Two initial solutions are obtained; one from a classical screening curve model, and another from a model using mixed integer linear programming (MILP). These initial solutions are perturbed and combined with an operational model to validate and further improve the solution. The developed model complements other models available from the literature, in its level of detail (power plant level and full year - hourly time resolution) combined with a fast computation time $(<1$ h). The evolution of the optimal amount of generation capacity as a function of the installed wind capacity is examined in a case study. As the share of wind power increases in the portfolio, a shift takes place from base load generation towards mid and peak load. This shift is triggered both by the lower demand and the increasing volatility. This demonstrates that operational constraints of power plants (individual basis) have an important impact on the configuration of the optimal generator set, and need being considered, especially at increasing rates of intermittent renewables.
\end{abstract}

Key-words: Power system planning; renewables; screening curve; unit commitment; wind power. 


\section{Nomenclature}

Sets

I (index i) Set of power plants

$\mathrm{J}$ (index $\mathrm{j}$ ) Set of time steps

$L$ (index I) Set of cost segments

$P$ (index $p) \quad$ Set of off line time steps

$\mathrm{T}$ (index $\mathrm{t}$ ) Set of technologies

\section{Parameters}

$\begin{array}{ll}A_{i} & \text { Fuel cost at minimum output [€/h] } \\ a_{i} & \text { Constant coefficient of quadratic cost function }[€ / \mathrm{h}] \\ b_{i} & \text { First order coefficient of quadratic cost function }[€ / \mathrm{MW} / \mathrm{h}] \\ c_{i} & \text { Second order coefficient of quadratic cost function }\left[€ / \mathrm{MW}^{2} / \mathrm{h}\right] \\ D_{j} & \text { Demand [MW] } \\ F_{i, l} & \text { Slope of linearized cost function [€/MWh] } \\ f c r_{t} & \text { Relative fixed cost [€/MW/y] } \\ M D T_{i} & \text { Minimum down time [h] } \\ M U T_{i} & \text { Minimum up time [h] } \\ O M_{i} & \text { Variable operation and maintenance cost [€/MWh] } \\ P \max _{i} & \text { Maximum power output [MW] } \\ P \min _{i} & \text { Minimum power output [MW] } \\ R_{j} & \text { System reserve [MW] } \\ S C_{i, p} & \text { Startup cost if offline for } p \text { hours [€] } \\ T_{i, l} & \text { Upper bound power limit of each segment [MW] } \\ T P & \text { Time period length, equal to } 1[\mathrm{~h}] \\ v c r_{t} & \text { Relative variable cost [€/MWh] }\end{array}$

Variables

$\begin{array}{ll}\delta(i, j, l) & \text { Generated electric power in each segment [MW] } \\ \operatorname{cap}(t) & \text { Installed capacity }[\mathrm{MW}] \\ f c & \text { Fixed cost }[€] \\ f u(i, j) & \text { Fuel cost }[€ / \mathrm{h}] \\ g(t, j) & \text { Hourly generation }[\mathrm{MW}] \\ o m(i, j) & \text { Variable operation and maintenance cost }[€ / \mathrm{h}] \\ s c(i, j) & \text { Startup cost }[€] \\ t c & \text { Total cost }[€] \\ u(i) & \text { Investment status }[-] \\ v c & \text { Variable cost }[€] \\ z(i, j) & \text { Commitment status }[-]\end{array}$




\section{Introduction}

Worldwide, electricity generation systems are undergoing major changes. The share of renewable energy sources (RES) is growing significantly, mainly driven by growing concerns on global warming and for reasons of strategic energy security. These RES, however, often have an intermittent profile. Their output is predictable only to a limited extent, and it is variable, not or only in a limited way dispatchable (e.g., wind turbines can be curtailed, reducing their output). The impact of these RES on the system is twofold: first, they reduce the residual load (i.e., the original load with RES generation subtracted). Second, more flexibility is required to deal with the higher variability of the residual load. Hence, these effects need to be accounted for in power system optimization [1]-[3].

Planning and operating of modern electric power systems comprehends several complex and interlinked tasks. These tasks can be divided in three main groups, depending on the considered time horizon. A first group includes long-term resource and equipment planning which targets time ranges from one year to several decades. Examples are investment planning, transmission and distribution planning and long range fuel planning. A second group contains short-term operational scheduling and is used for time intervals from several hours to a few weeks, or even year(s). Examples are unit commitment (UC) scheduling, maintenance and production scheduling and fuel scheduling. The last group includes real time operations, which consider fractions of a second to several minutes.

Examples of real time operations are automatic protection and dispatching. The focus of the present paper is on the first and second group of models.

The main research questions of this paper are (1) to what extent does the deployment of RES affect the optimal generation mix, and (2) to what extent do technical operational constraints need being accounted for in system planning models, to achieve meaningful and reliable results. Hence, in this paper, a new electricity generation system planning model is developed, focusing on the integration of RES, and the corresponding required flexibility of dispatchable generation. The objective is to integrate operational constraints from short-term generation scheduling models into a power system planning model. Specific focus is on the impact of RES on lowering the residual demand on the one hand, and on increasing the need for flexibility by a more variable residual load profile, on the other hand (both creating a shift from base to mid load and peak load). The developed model will further allow evaluating classic system planning approaches, which are also often used as such in this context.

Several power system planning models have been developed over time [4]. While some focus on the expansion of the transmission and/or distribution system [5][6], the scope of this work is on expansion of the electricity generation system. The textbook example of generation system planning optimization is the so-called "load duration based" or "screening curve" approach (see, e.g., Stoft[7]). Several specific features have been added to such planning models. Santos and Legey incorporate environmental costs in an expansion planning model [8]. Delgado et al. use a planning model in a stochastic setting to focus on the impact of nuclear (they for instance consider different scenarios for fuel and allowance prices) [9]. Unsihuay-Vila et al. consider both generation and transmission expansion planning, in a load duration based model setting with multiple objectives (i.e., cost, lifecycle emissions and diversification) [10].

RES integration (e.g., wind) is often studied from an operational viewpoint (see, e.g., [11][12]). However, also from a planning perspective, the flexibility requirement in modeling is being 
acknowledged [13]. Recent developed models for system planning made efforts to take explicitly into account the flexibility limitations of thermal generators, focusing on wind power integration, preserving the chronological order of time segments (hourly or aggregated) [14][15]. Delarue et al. also added uncertainty on cost parameters as risk in such framework [16]. Also in existing bottom-up modeling frameworks, such as ReEDS [17] and TIMES [18][19], the impact of RES are being studied. These models are typically set-up as linear models, working on technology rather than individual power plant basis, and considering a limited set of so-called time-slices, to represent the variations in demand and RES generation. Within such frameworks, efforts have recently been made to expand the temporal resolution, to cover the full 8760 hours [20]. Such improved representation can have significant impact on the actual amount of RES generation achieved in a system or on $\mathrm{CO}_{2}$ emissions [21][22]. Several approaches also have used planning models as TIMES, soft-linked to an operational model to validate results in technical terms [23][24].

Other models start from an operational model and include the investment decision as a variable, taking into account a limited number of time periods [25]. A recent formulation/analysis, combining full year representation and power plant resolution has been presented by Palmintier [26][27]. A clustered UC formulation is proposed, embedded in a planning model formulation.

Compared to the existing literature, this paper presents a novel approach for system planning optimization. It takes a full year horizon (with hourly time steps) into account and works on a power plant resolution (rather than on a technology basis), taking into account technical operational constraints (with binary variables). The developed modeling methodology can be solved in relatively limited calculation time $(<1 \mathrm{~h})$. It can therefore easily be used for broader system studies and sensitivity analyses. This model is then used to quantify the impact of taking into account technical constraints of power plants, and as such, addresses the impact of intermittent RES on electricity generation planning. This way, the present research adds to the available literature, by the development of the model and the quantitative insights stressing the relevance of operational characteristics.

The structure of this paper is as follows. The next section presents the problem formulation, first for the power system planning model (on technology basis), second for an operational optimization (UC) and third for an integrated approach. Section 3 presents the newly developed methodology. In section 4 the simulation results of the case study are presented and discussed. Section 5 concludes this paper.

\section{Problem formulation}

Many models exist to optimize the electricity system. The models relevant in the context of this paper can be traditionally divided in several groups (see discussion in previous section), i.e., the system planning models, the operational scheduling models or a combined approach. A problem formulation of each model type will be elaborated below.

\subsection{System planning}

The objective of this model type is to determine the optimal mix of generation technologies able to meet a given load. The mix of generation technologies has to be optimal in a cost effective way, 
based on fixed and variable costs. The technology specific fixed cost is an annualized cost [ $€ / \mathrm{MW} / \mathrm{y}]$, covering investment as well as fixed operation and maintenance costs. The technology specific variable cost [ $€ / \mathrm{MWh}]$ encompasses fuel and variable operational and maintenance costs.

A system planning model can be formulated as linear program (LP) which uses only continuous variables. The solution of the model is expressed as an amount of generation capacity for each generation technology. The lack of discrete variables implies that no discrete number of generation units can be calculated. Furthermore no inter-hourly constraints are taken into account (as there is no resolution on power plant level). The time horizon of a system planning method is at least one year.

The objective of a basic system planning model is to minimize the overall cost $t c$ [ $€$ ] (for a one year period). This cost is equal to the sum of the fixed cost $f c[€]$ and variable cost $v c[€]$ :

$$
\text { Minimize tc }=f c+v c
$$

The fixed cost is the sum of the fixed costs of the different installed technologies $t$ (set $T$ ), with $f c r$ the relative fixed cost for each technology [€/MW/y] and cap the installed capacity [MW]:

$$
f c=\sum_{t} f c r_{t} \cdot \operatorname{cap}(t)
$$

The variable cost is the sum of the variable cost over all technologies $t$ and all time periods $j$ (every hour of the year), with $v c r$ the relative variable cost [ $€ / M W h]$, and $g$ the hourly generation [MW] (TP is the operational time period length, equal to $1[\mathrm{~h}])$ :

$$
v c=\sum_{t, j} v c r_{t} \cdot g(t, j) \cdot T P
$$

For every hour $j$, the total generation needs to meet the demand $D$, while the hourly generation per technology is restricted by the installed capacity:

$$
\begin{aligned}
& \sum_{t} g(t, j)=D_{j}, \quad \forall j \in J \\
& g(t, j) \leq \operatorname{cap}(t), \quad \forall t \in T, \forall j \in J
\end{aligned}
$$

The optimization of this basic model corresponds to obtaining a solution through the so-called screening curve methodology [14].

\subsection{Short-term operational scheduling model}

The objective of this model type is to determine the optimal operational scheduling for a given set of generation units. The portfolio of power plants is fixed and not part of the optimization. Compared to the operational part of the system planning model, this model works on power plant basis (compared to technology) and has much greater technical detail.

The operational cost calculated by these models is composed of the fuel cost and startup cost. A minimum amount of upward and downward spinning reserve, a minimum up and down time for each generation unit and a minimum generation output (if online) for each unit is enforced. 
A cost driven UC optimization problem is considered. This problem has been described widely in the literature. The description as presented below is partly based on [28].

The objective to be minimized is the total generation cost $v c$, which is equal to the sum of fuel costs $f u$, startup costs $s c$ and variable O\&M cost om, over all power plants $i$ and all time periods $j$ (in this case hourly time steps):

$$
\text { minimize } v c=\sum_{i, j} f u(i, j) \cdot T P+\sum_{i, j} s c(i, j)+\sum_{i, j} o m(i, j) \cdot T P
$$

The fuel cost of a power plant is typically a quadratic function of its output $g$ and commitment status $z$ (with $a, b$ and $c$ the cost coefficients) [29]:

$$
f u(i, j)=a_{i} \cdot z(i, j)+b_{i} \cdot g(i, j)+c_{i} \cdot g(i, j)^{2}, \quad \forall i \in I, \forall j \in J
$$

This (convex) quadratic cost function can be linearized by a number of stepwise linear segments (index I). Let Pmax and Pmin be the maximum and minimum power output (if online) respectively, $A$

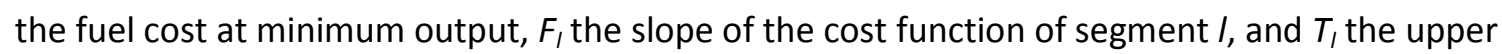
bound power limit of each segment (note that in this case for the last segment $n l: T_{n l}=$ Pmax). With $\delta$ the actual power generation in each segment $I$, the fuel cost and generation limits are set by the following equations:

$$
\begin{gathered}
f u(i, j)=A_{i} \cdot z(i, j)+\sum_{l} F_{i, l} \cdot \delta(i, j, l), \quad \forall i \in I, \forall j \in J \\
g(i, j)=\operatorname{Pmin}_{i} \cdot z(i, j)+\sum_{l} \delta(i, j, l), \quad \forall i \in I, \forall j \in J \\
g(i, j) \leq \operatorname{Pmax}_{i} \cdot z(i, j), \quad \forall i \in I, \forall j \in J \\
\delta(i, j, l) \leq T_{i, l}-T_{i, l-1}, \quad \forall i \in I, \forall j \in J, \forall l=2 \ldots n l \\
\delta(i, j, l) \leq T_{i, l}-\operatorname{Pmin}_{i}, \quad \forall i \in I, \forall j \in J, l=1 \\
\delta(i, j, l) \geq 0, \quad \forall i \in I, \forall j \in J, \forall l \in L
\end{gathered}
$$

Parameter $A_{i}$ is the cost [ $\left.€ / \mathrm{h}\right]$ at minimum output, and determined as

$$
A_{i}=a_{i}+b_{i} \cdot \operatorname{Pmin}_{i}+c_{i} \cdot \operatorname{Pmin}_{i}^{2}, \forall i \in I, \forall j \in J
$$

The commitment status $z$ is a binary variable:

$$
z(i, j) \in\{0,1\}, \forall i \in I, \forall j \in J
$$

The startup cost is a function of the time $p$ the plant has been off line, previous to the startup. This is implemented as follows, with parameter $S C_{p}$ presenting the startup cost if previously shut down for $p$ hours (set $P$ ):

$$
\begin{gathered}
s c(i, j) \geq S C_{i, p} \cdot\left[z(i, j)-\sum_{n=1}^{p} z(i, j-n)\right], \quad \forall i \in I, \forall j \in J, \forall p \in P \\
s c(i, j) \geq 0, \quad \forall i \in I, \forall j \in J
\end{gathered}
$$

The $O \& M$ cost is a linear function of the output, with $O M$ the variable $O \& M$ cost:

$$
o m(i, j)=O M_{i} \cdot g(i, j), \quad \forall i \in I, \forall j \in J
$$


During all time periods $j$, the sum of the power generated $g$ of all the power plants should be equal to the demand $D_{j}$ :

$$
\sum_{i} g(i, j)=D_{j}, \quad \forall j \in J
$$

Furthermore, a certain amount of system reserves $R_{j}$ need to be present in the system, both up- and downwards:

$$
\begin{aligned}
& \sum_{i} \operatorname{Pmax}_{i} \cdot z(i, j) \geq D_{j}+R_{j}, \quad \forall j \in J \\
& \sum_{i} \operatorname{Pmin}_{i} \cdot z(i, j) \leq D_{j}-R_{j}, \quad \forall j \in J
\end{aligned}
$$

Finally, the minimum up and down times (MUT and MDT, respectively) are imposed as follows:

$$
\begin{aligned}
& z(i, j)-z(i, j-1)-z(i, j+n) \leq 0, \quad \forall i \in I, \forall j \in J, \forall n=1 \ldots M U T_{i}-1 \\
& z(i, j-1)-z(i, j)+z(i, j+n) \leq 1, \quad \forall i \in I, \forall j \in J, \forall n=1 \ldots M D T_{i}-1
\end{aligned}
$$

The operational optimization problem consists of the objective (6) and the constraints (8)-(23).

\subsection{Integrated model}

The above discussed models can now be combined, to include investment decisions (system planning) on the one hand, as well as a resolution on generation unit level and high technical detail, on the other hand. Towards this end, the system planning model is adjusted to run on power plant level (discrete generation units - index $i$ ) instead of technology level (index $t$ ). An additional binary variable $u$ is introduced to represent the investment decision in certain unit $i$. The initial set $l$ of power plants should be sufficiently expanded, to include enough (possible) units of each technology, to make an actual optimization possible, also in planning.

$$
f c=\sum_{i} f c r_{i} \cdot P \max _{i} \cdot u(i)
$$

The following constraints further need to be set on $u$ :

$$
\begin{aligned}
& u(i) \geq z(i, j), \quad \forall i \in I, \forall j \in J \\
& u(i) \in\{0,1\}, \quad \forall i \in I
\end{aligned}
$$

The variable cost of the system planning model (Eq. (3)) is replaced by the more detailed variable cost of the operational model, as defined in Eq. (6). Hence, the integrated model has objective function Eq. (1), and constraints (6), (8)-(26).

Such integrated model could in theory determine the optimal set of generation units [30]. However, since the computational requirements increase exponentially with the time horizon, it is extremely difficult to solve the optimization problem for an entire year due to computational limitations. A typical solution for this problem is provided in section 3.2 below. 


\section{Integrated system planning model with operational constraints}

In this section, a new modeling approach is described, allowing to optimize the integrated system planning model (as described in Section 2.3), incorporating technical constraints with an hourly and power plant level resolution, in an acceptable computation time.

\subsection{Overall methodology}

The overall model consists of different sub-models. First an initial set of power plants is determined. Second, this initial set is used in combination with an operational model to first validate the solution in operational terms, and second to iteratively adjust the portfolio of power plants to move towards an optimal solution (perturbation algorithm together with operational UC model). An overview of this methodology is presented in Figure 1.

As shown in Figure 1, different system planning models are used to determine the initial set of power plants. The performance of each system planning model is discussed together with the simulation results in section 4 .

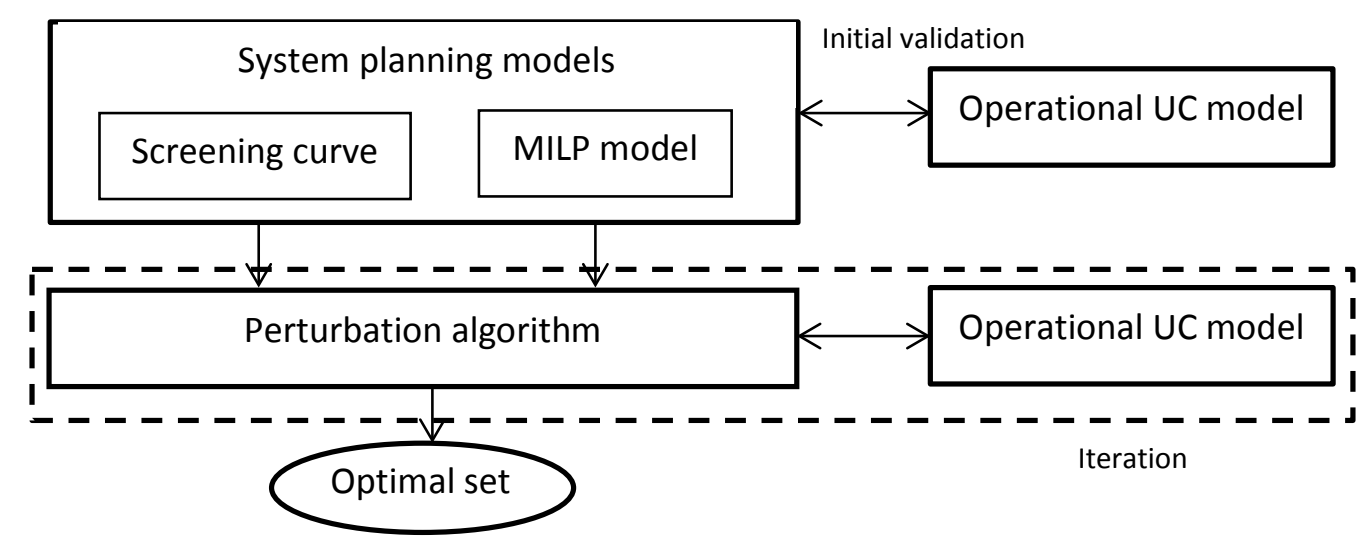

Figure 1. Overview of the developed methodology.

The different sub-models (system planning models, perturbation algorithm and operational UC model) are now discussed.

\subsection{System planning models}

In a first stage two different system planning approaches are developed and used, to obtain a power plant portfolio on generation unit level. This solution will then be validated in operational terms and can be assessed as such. Furthermore, this solution will also serve as a starting solution for further iterative improvement by the perturbation algorithm.

\section{Screening Curve model}

The first model is based on a basic screening curve model as presented before in section 2.1 (solved by linear programming). The demand considered in the model formulation is the original demand increased with the required reserves. The temporal resolution is one hour, and the horizon of this 
model is one year. The initial output of this screening curve model is a continuous amount of generation capacity. In order to use this result, it is converted to a discrete number of generation units. This is done by rounding-up the installed capacity per generation technology to the nearest multiple of the capacity of a single unit.

\section{MILP system planning model}

A second system planning model is an MILP model with the investment decision as an extra variable. This model is essentially a direct implementation of the integrated model as described earlier (objective Eq. (1), and constraints (6), (8)-(26)), as MILP.

As this problem formulation is too complex to solve for a time horizon of an entire year, a typical approach is to use a set of representative days/weeks (see, e.g., [25]). These different representative periods (days up to weeks) can each be modeled in a cyclic way, and thus are decoupled in operational terms. The investment variables, however, are set over all representative periods as a whole. In the model simulations, a set of three representative days will be used. A methodological illustration is presented in Figure 2.

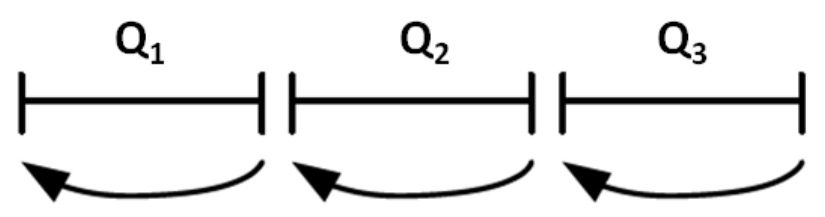

Figure 2. Cyclic linkage of three representative periods.

It is important to capture the fluctuations in demand during the year. Hence, the three time periods are selected as the day that contains the minimum demand level of the year, the day that contains the maximum demand level and the day that contains the largest demand spread in 24 hours. The importance of each time period is weighted in such way that the weighted sum of the hourly demand of the three selected days equals the overall original demand of the entire year.

\subsection{Operational UC model}

The operational model formulation corresponds to Eq. (6) and (8)-(23), as presented earlier. The model used in the overall algorithm is based on an enhanced priority list approach, as developed by Delarue et al. [31]. As demonstrated by these authors, this UC model is well suited to solve relatively large instances (one year period, hourly time steps) with good accuracy in short computation times. Furthermore, this model is well suited to deal with low residual demand problems (which is a relevant feature in the present framework, i.e., low residual demand because of RES penetration) ${ }^{1}$. First, this model is used to validate the original sets in terms of operational feasibility. Second, this model is used in combination with the perturbation algorithm (see next subsection 3.4 ) to iteratively improve the solution.

\footnotetext{
${ }^{1}$ Alternatively, an operational MILP model could be used, solving the year in sequential blocks (as solving one year as whole is too difficult in computational terms). However, the enhanced priority list method is preferred, mainly because of its computational efficiency.
} 


\subsection{Perturbation algorithm}

The original generator sets determined by the two system planning models can be perturbed (i.e., search in the immediate vicinity of these initial estimated sets) to find a more optimal set. The perturbation algorithm is composed of two parts. Both parts require the use of the operational UC model (discussed in the previous subsection 3.3), to validate a certain (intermediate) solution.

The first part of the perturbation algorithm aims to subtract units from the estimated set. In every iteration, a single unit is subtracted. To determine which unit is subtracted, the algorithm subsequently removes temporarily a unit of each generation type and calculates the total cost of the reduced sets. This total cost consists of the fixed costs (related to the set of power plants) and the variable cost determined by running the operational UC model. The generation type that leads to the largest cost reduction when subtracted, is permanently removed from the set of generation units. A schematic overview is shown in Figure 3.

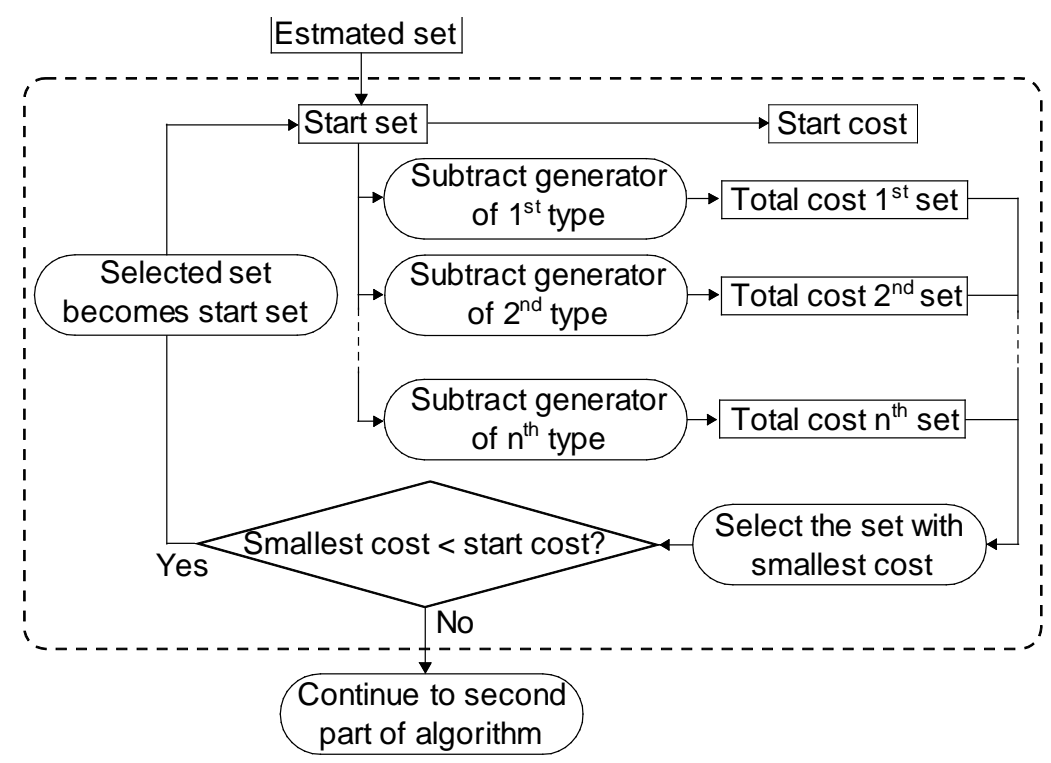

Figure 3. Schematic representation of the first part of the perturbation algorithm.

The first part of the algorithm is completed when no further cost reduction can be achieved by removing a generation unit. The second part of the algorithm is analogous to the first part. This time a unit is added in each iteration.

Note that when a unit is subtracted during an iteration, it is often not possible to serve the demand with the remaining generator set. Therefore, other units are simultaneously added when one unit is subtracted. The units that are added have a combined capacity which is less than the capacity of the subtracted unit. An analogous remark can be made for the second part of the algorithm, i.e., when adding a unit, it is usually possible to turn off a more expensive unit for the entire time horizon. In this case the adding of one unit will lead to the simultaneous removal of another unit. 


\section{Model simulations}

The developed model is applied to a methodological case study, optimizing the planning of an electricity generation system, under different wind deployment scenarios. The next subsection describes the input data, while the following subsection presents an overview of the model outcome. A third subsection discusses the performance of the different system planning models compared to the overall algorithm. Finally, the fourth subsection focuses on the evolution of the system with increasing amounts of wind generation.

\subsection{Data description}

The demand profile is based on load-data from the Belgian transmission system operator Elia [32] for the year 2011. The magnitude is scaled and the resolution of the load-data is modified to obtain a demand profile with load values at hourly intervals and a maximum load of approximately $8 \mathrm{GW}$. The time horizon of the demand profile is one year. The reserve requirement is set at $5 \%$ of the demand, in both upward and downward direction.

The selected profile of electricity generated from wind energy is based on Belgian generation data from 2011. The generation data is obtained from Elia [33]. Analogously to the demand profile, the resolution of the generation profile is converted from quarter values to hourly values. The magnitude of the generation profile is varied linearly to create five different scenarios. The amount of wind capacity installed in each scenario ranges from minimum $0 \mathrm{MW}$ (wind scenario A) to maximum 4334 $\mathrm{MW}$ (wind scenario $\mathrm{E}$ ). This maximum amount of installed wind results in a residual demand with a minimum of $150 \mathrm{MW}$ and $26 \%$ of the electricity demand being covered by wind.

Table 1 displays a number of characteristics for every scenario, from wind scenario $A$ to $E$. The amount of installed wind capacity in each scenario is presented. The residual demand for each scenario is characterized by the minimum, maximum and average load. At last two key figures are displayed to quantify the volatility of the demand in each scenario. The first number is the maximum spread (i.e., the maximum demand minus the minimum demand) that occurs in a 24 hour time period. A second number given is the average diurnal spread relative to the annual peak demand, referred to as the average diurnal spread.

Table 1. Characteristics of the residual demand and wind profile of each scenario.

\begin{tabular}{llllll}
\hline Wind scenario & A & B & C & D & E \\
\hline Wind cap [MW] & 0 & 1083 & 2167 & 3250 & 4334 \\
Wind energy [\%] & 0 & 6.5 & 13 & 19.5 & 26 \\
\hline Min load [MW] & 3786 & 3150 & 2254 & 1206 & 150 \\
Max load [MW] & 7881 & 7879 & 7877 & 7874 & 7872 \\
Avg load [MW] & 5712 & 5339 & 4965 & 4592 & 4219 \\
\hline Max 24h spread [MW] & 2713 & 2991 & 3733 & 4535 & 5339 \\
Average diurinal spread & $20.98 \%$ & $22.00 \%$ & $24.47 \%$ & $27.93 \%$ & $31.97 \%$ \\
\hline
\end{tabular}

A set of five different generation technologies is selected to represent potential generating units. The economical characteristics of each generation technology are based on data obtained from the 
IEA/NEA Projected Costs of Generating Electricity study (2010 edition) [34]. The technical characteristics are based on data obtained from Kazarlis et al. [35] and Grigg et al. [36]. Note that these characteristics are purely methodological and intended to represent different types of generation technology (i.e., base, mid and peak load) and thus, are to be regarded as such.

The technical data is shown Table 2. tcold represents the amount of hours after the minimum downtime from when it is assumed that the unit will make a cold start. A last characteristic given is $L T$, the assumed lifetime of each generator type.

Table 2. Reference set of generation technology: technical data.

\begin{tabular}{|c|c|c|c|c|c|c|}
\hline Technology & $\begin{array}{l}\text { Pmin } \\
\text { [MW] }\end{array}$ & $\begin{array}{l}\text { Pmax } \\
\text { [MW] }\end{array}$ & $\begin{array}{l}\text { MUT } \\
\text { [h] }\end{array}$ & $\begin{array}{l}\text { MDT } \\
{[\mathrm{h}]}\end{array}$ & $\begin{array}{l}\text { tcold } \\
{[\mathrm{h}]}\end{array}$ & $\begin{array}{l}\text { LT } \\
\text { [y] }\end{array}$ \\
\hline Oil & 10 & 50 & 1 & 1 & 0 & 30 \\
\hline Peak Gas & 30 & 150 & 1 & 2 & 1 & 30 \\
\hline CCGT & 147 & 420 & 2 & 4 & 2 & 30 \\
\hline Coal & 300 & 750 & 5 & 8 & 4 & 30 \\
\hline Nuclear & 600 & 1000 & 8 & 24 & 8 & 40 \\
\hline
\end{tabular}

Table 3 displays all cost-related characteristics. The investment cost is denoted by INV, and needs to be converted to an annual cost $f c r$ by using the lifetime $L T$. A two-step startup cost function is considered for $S C_{i, p}$, with $h c$ and $c c$ representing the hot and cold startup cost, respectively (set $P$ ).

Table 3. Reference set of generation technology: cost data.

\begin{tabular}{|c|c|c|c|c|c|c|c|}
\hline Technology & $\begin{array}{l}\text { INV } \\
{[€ / k W]}\end{array}$ & $\begin{array}{l}a \\
{[€ / h]}\end{array}$ & $\begin{array}{l}\mathrm{b} \\
{[€ / \mathrm{MW} / \mathrm{h}]}\end{array}$ & $\begin{array}{l}\mathrm{c} \\
{\left[€ / \mathrm{MW}^{2} / \mathrm{h}\right]}\end{array}$ & $\begin{array}{l}\text { hc } \\
{[€]}\end{array}$ & $\begin{array}{l}\text { CC } \\
{[€]}\end{array}$ & $\begin{array}{l}\text { OM } \\
\text { [€/MWh] }\end{array}$ \\
\hline Oil & 461 & 450 & 104 & 0.034 & 30 & 60 & 18 \\
\hline Peak Gas & 720 & 950 & 65.48 & 0.0043 & 1898 & 3796 & 5.38 \\
\hline CCGT & 1069 & 1320 & 49.65 & 0.001 & 3765 & 7530 & 4.06 \\
\hline Coal & 2539 & 2100 & 22.8 & 0.0002 & 5632 & 11264 & 8.73 \\
\hline Nuclear & 5383 & 3500 & 12.33 & 0.0001 & 7500 & 15000 & 7.2 \\
\hline
\end{tabular}

\subsection{Simulation results}

The main model is simulated for each scenario. Each simulation comprises solving for the initial set by the two system planning models, together with a perturbation of these estimations. The operational cost is determined with the operational UC model. All optimizations with the MILP system planning model are run using an optimality gap of $1 \%$. The model is solved on an Intel E8400 Duo core at $3 \mathrm{GHz}$, with $4 \mathrm{~GB}$ RAM memory. 
The simulation results for each scenario are displayed in Table 4 . The set of generation units calculated by each system planning model is displayed together with the optimal set determined after perturbation. An investment cost and the operational cost of serving the residual demand is calculated for each set. The sum of investment and operational cost is shown as the total cost. The relative gap denotes the difference between the total cost of each original generator set before perturbation and the total cost of the optimal set as a percentage of the total cost of the optimal set. A last result given is the number of iterations needed by the perturbation algorithm to reach the optimal solution. In some cases the perturbation of an estimated set converges to a non-optimal set. This is indicated by 'NOC' (Non-Optimal Convergence).

Table 4. Simulation results for the different wind scenarios (A - E). SC denotes the set of power plants obtained with the screening curve methodology, MILP denotes the MILP system planning model (representative days), while Optimal denotes the set obtained after perturbation.

\begin{tabular}{|c|c|c|c|c|c|c|c|c|c|}
\hline wind scenario & & A & & & B & & & C & \\
\hline model & SC & MILP & Optimal & SC & MILP & Optimal & SC & MILP & Optimal \\
\hline Oil [\#] & 14 & 5 & 20 & 20 & 7 & 25 & 23 & 8 & 28 \\
\hline Peak Gas [\#] & 3 & 0 & 3 & 3 & 2 & 3 & 4 & 1 & 2 \\
\hline CCGT [\#] & 2 & 2 & 2 & 2 & 2 & 2 & 2 & 2 & 2 \\
\hline Coal [\#] & 1 & 3 & 0 & 1 & 4 & 1 & 2 & 4 & 1 \\
\hline Nuclear [\#] & 6 & 5 & 6 & 6 & 4 & 5 & 6 & 4 & 5 \\
\hline Operational cost [M€] & 1227.34 & 1276.43 & 1265.19 & 1137.13 & 1250.06 & 1217.07 & 1058.46 & 1147.7 & 1118.32 \\
\hline Investment cost $[\mathrm{M} €]$ & 922.41 & 897.07 & 863.55 & 927.02 & 834.71 & 796.29 & 996.4 & 831.88 & 795 \\
\hline Total cost [M€] & 2149.75 & 2173.51 & 2128.74 & 2064.16 & 2084.77 & 2013.36 & 2054.87 & 1979.58 & 1913.31 \\
\hline Relative gap & $0.98 \%$ & $2.06 \%$ & $0.00 \%$ & $2.46 \%$ & $3.43 \%$ & $0.00 \%$ & $6.89 \%$ & $3.35 \%$ & $0.00 \%$ \\
\hline Perturbation steps [\#] & 5 & NOC & - & 4 & NOC & - & 5 & NOC & - \\
\hline wind scenario & & $\mathrm{D}$ & & & E & & & & \\
\hline model & SC & MILP & Optimal & SC & MILP & Optimal & & & \\
\hline Oil [\#] & 26 & 16 & 30 & 28 & 12 & 36 & & & \\
\hline Peak Gas [\#] & 4 & 0 & 3 & 4 & 1 & 4 & & & \\
\hline CCGT [\#] & 2 & 3 & 2 & 3 & 2 & 2 & & & \\
\hline Coal [\#] & 2 & 5 & 2 & 2 & 9 & 3 & & & \\
\hline Nuclear [\#] & 5 & 3 & 4 & 5 & 0 & 3 & & & \\
\hline Operational cost [M€] & 1017.09 & 1130.87 & 1098.01 & 955.1 & 1303.59 & 1096.21 & & & \\
\hline Investment cost [M€] & 864.13 & 778.29 & 729.03 & 880.64 & 614.03 & 666.14 & & & \\
\hline Total cost [M€] & 1881.22 & 1909.16 & 1827.04 & 1835.74 & 1917.61 & 1762.35 & & & \\
\hline Relative gap & $2.88 \%$ & $4.30 \%$ & $0.00 \%$ & $4.00 \%$ & $8.10 \%$ & $0.00 \%$ & & & \\
\hline Perturbation steps [\#] & 6 & NOC & - & NC & NOC & - & & & \\
\hline
\end{tabular}

These results are now discussed, first in terms of modeling performance, and second focusing on the outcome, regarding the impact of increasing intermittent RES on the rest of the system. 


\subsection{Performance of the different planning models}

The results presented in Table 4 are now further analyzed. A first question is whether the set of power plants obtained with a basic system planning model (that does not take into account detailed technical constraints (screening curve) or only considers a limited time frame (MILP)), is actually feasible to serve the load over the entire year, with technical constraints taken into account (solved with the operational model). As it turns out, the initial sets are feasible in operational terms in all simulations (both models, all wind scenarios). When comparing the initial sets of both models, it can be seen that the amount of generation capacity determined by the screening curve model (SC) is larger than the amount of capacity determined by the MILP model. This is explained by the fact that the screening curve model rounds up the optimal amount of generation capacity to convert the amount of capacity to an amount of discrete generation units. A small amount of capacity needed can thereby lead to the installation of an entire generation unit ${ }^{2}$. Despite the higher installed capacity, the total cost of the screening curve model is in every wind scenario lower than the MILP model.

When using the initial sets of the two planning models in the perturbation algorithm, in every wind scenario the final solution obtained with the screening curve outperforms the solution obtained when starting from the MILP model. This best solution is denoted "optimal" and each time presented as third solution. The final solution obtained from the MILP after perturbation is $0.36 \%, 1.3 \%, 0.53 \%$, $1.15 \%$ and $5.39 \%$ higher than the best solution (obtained from starting perturbation from the SC set), for wind scenario $A, B, C, D$ and $E$, respectively. There is further a trend of an increasing difference between the overall cost of the initially estimated sets and that of the optimal set, as wind power increases.

A note needs to be made on the perturbation of the initial set of the screening curve model in wind scenario E. Perturbing this estimated set leads not to convergence. During an iteration of the perturbation process, a set was obtained, for which it was not possible to determine a feasible commitment schedule. This is indicated as ' $\mathrm{NC}$ ' (No Convergence) in Table 4. Hence, the initial set was modified in a heuristic manner, removing one CCGT unit and adding peak oil fired units instead. With this solution as start value for the perturbation algorithm, the optimal set was obtained. Perturbing the estimated set determined by the MILP system planning model did converge but again to a non-optimal set.

It can be concluded that using the developed methodology with the screening curve method to determine the initial set, is the preferred way to set up and use the model. Overall computation times in this set up stay below $1 \mathrm{~h}$ for each scenario. The numerical results show that the screening curve model provides a better estimation of the optimal generator set compared to the MILP system planning model. The screening curve model provides a quick estimation of the optimal set, but perturbation is always needed. The results obtained from the MILP system planning model are always far from the optimal generator set. Perturbation of these results thereby does not lead to the optimal set of generators. In addition the set estimated by the MILP model differs strongly for each

\footnotetext{
${ }^{2}$ Another way to convert the continuous amount of generation capacity to a discrete number of units is to round one type of generation capacity to the nearest number of discrete units and add the difference in generation capacity obtained to the generation capacity of another type of technology. This method is examined, but the resulting generator set is generally not able to serve the demand. This method is therefore not further pursued.
} 
scenario. This is explained by the fact that the MILP model makes its investment decision on a very limited amount of data.

As outlined in section 3.2, the MILP system planning model works with the days containing the maximum and minimum demand and a day with the largest demand spread. The relative importance of each representative day is incorporated through different weights in the objective function. This way, the minimum, maximum and average demand are equal for the whole demand and the representative profile ( 3 days). However, these numbers cannot characterize the entire demand profile. As an example, the relative average diurnal spread is computed for both the entire demand profile and the three representative days. This is an indication for the volatility of the demand. A number for each scenario is shown in Table 5 . It is clear that the amount of volatility differs significantly. As other relevant characterizing parameters could be identified as well, it is not possible to capture all of these in the right proportion. Especially the variable profile of wind is difficult to capture in representative days, even more when combined with the fluctuating demand ${ }^{3}$. Hence, this indicates the necessity to consider both demand and RES on a time frame sufficiently large. In this case, using 3 days clearly is insufficient to achieve an adequate representation.

Table 5. Indication of the difference in volatility between the entire demand profile and the representative days.

\begin{tabular}{llllll}
\hline Scenario & A & B & C & D & E \\
\hline Entire demand profile & $20.98 \%$ & $22.00 \%$ & $24.47 \%$ & $27.93 \%$ & $31.97 \%$ \\
Three days & $22.31 \%$ & $25.95 \%$ & $31.02 \%$ & $41.37 \%$ & $47.04 \%$ \\
\hline
\end{tabular}

\subsection{Evolution of the optimal generator set as a function of wind power deployment}

The influence of the amount of wind capacity on the estimated (screening curve and MILP) and optimal sets (after perturbation) of generation units is shown by Figure 4 . The figure displays the amount of installed capacity per generation type, per system planning model and per scenario.

\footnotetext{
${ }^{3}$ The original load as such could be characterized more easily, as it has typical diurnal, weekly and seasonal patterns.
} 


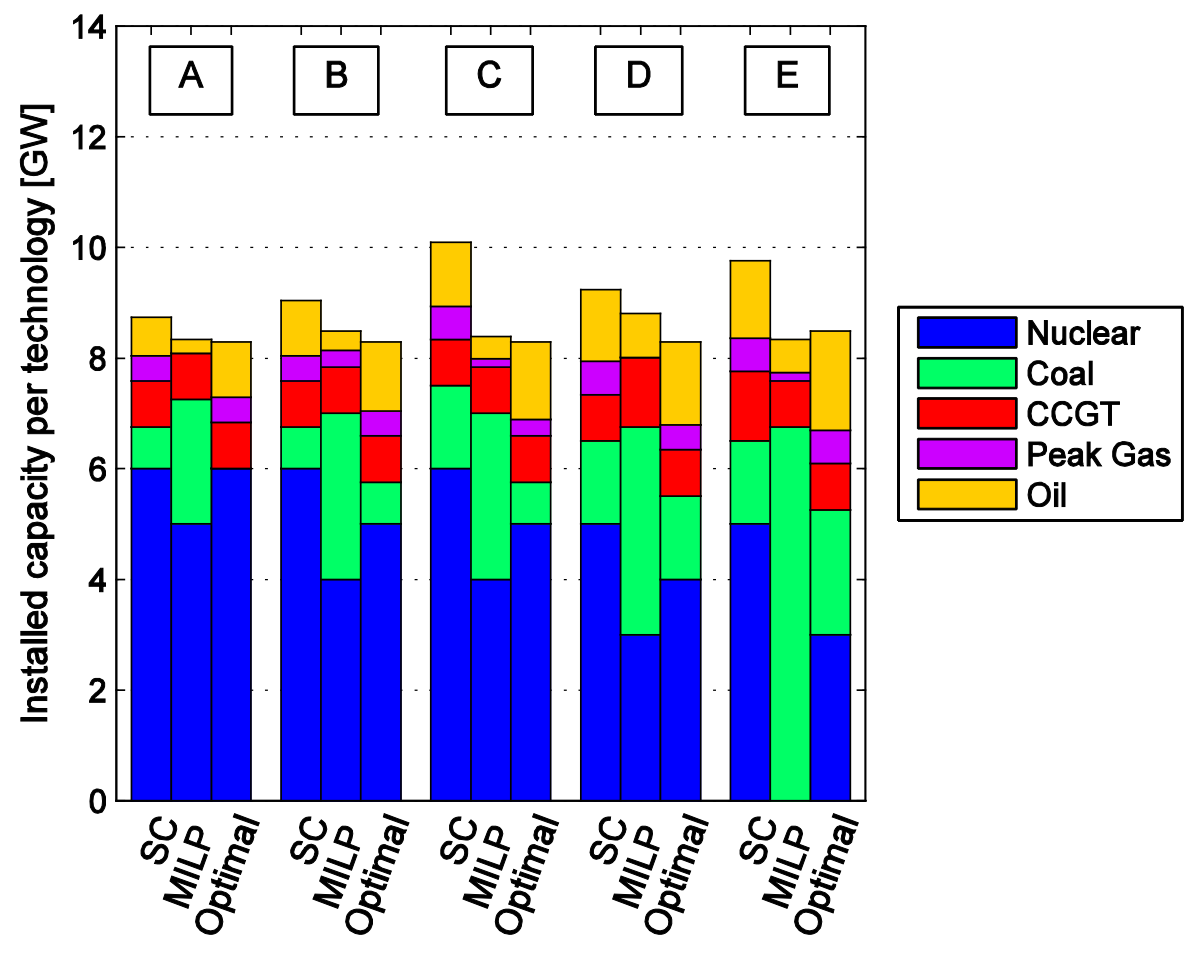

Figure 4. Installed capacity obtained as estimated set (screening curve SC and MILP) and after perturbation (optimal) for each scenario.

Figure 5 provides a more detailed illustration of the optimal generator set as a function of the wind capacity. It is clear that when more wind capacity is installed, the amount of base-load capacity reduces while the amount of mid-load and peak-load generation units increases. The total amount of installed capacity (excluding wind) remains approximately equal for all scenarios.

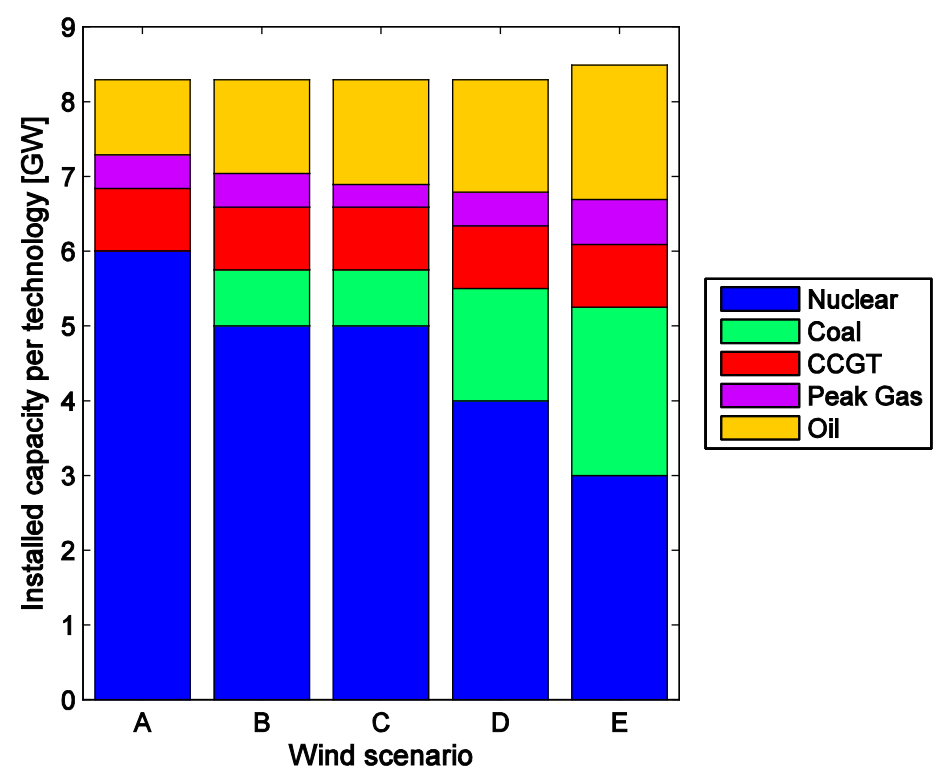

Figure 5. Installed capacity in optimal generator set for each scenario. 
The replacement of base-load capacity by mid- and peak-load capacity in scenarios with more wind, is explained by two different effects. The first effect is an economic effect. An increase of installed wind capacity causes the total residual demand to decrease. In turn, this leads to a decrease of the total output of each generation unit. The decrease of generation output is unfavorable for base-load generation units as they are only economically preferable when their generation output is high.

The second effect is an operational effect. An increasing amount of installed wind capacity causes an increasing volatility of the residual demand. An increasing amount of flexible generation capacity is required to serve a demand with increasing volatility. This is again unfavorable for the base-load generation units, since their operational flexibility is more limited than the flexibility of a peak-load plant.

A comparison between two sets of generation capacity is made to examine the importance of both effects. The first set is determined with the screening curve model without rounding the obtained solution to a discrete set of generation units. This implies that only the economic effect (reducing the demand) influences the amount of installed capacity in the first set. The second set is the optimal set of generators, as determined by the developed methodology, incorporating operational constraints. In this case, both the economical and operational effect influence the amount of installed capacity as a function of the installed wind capacity. The installed generation capacity in each set as a function of the wind capacity is shown in Figure 6.

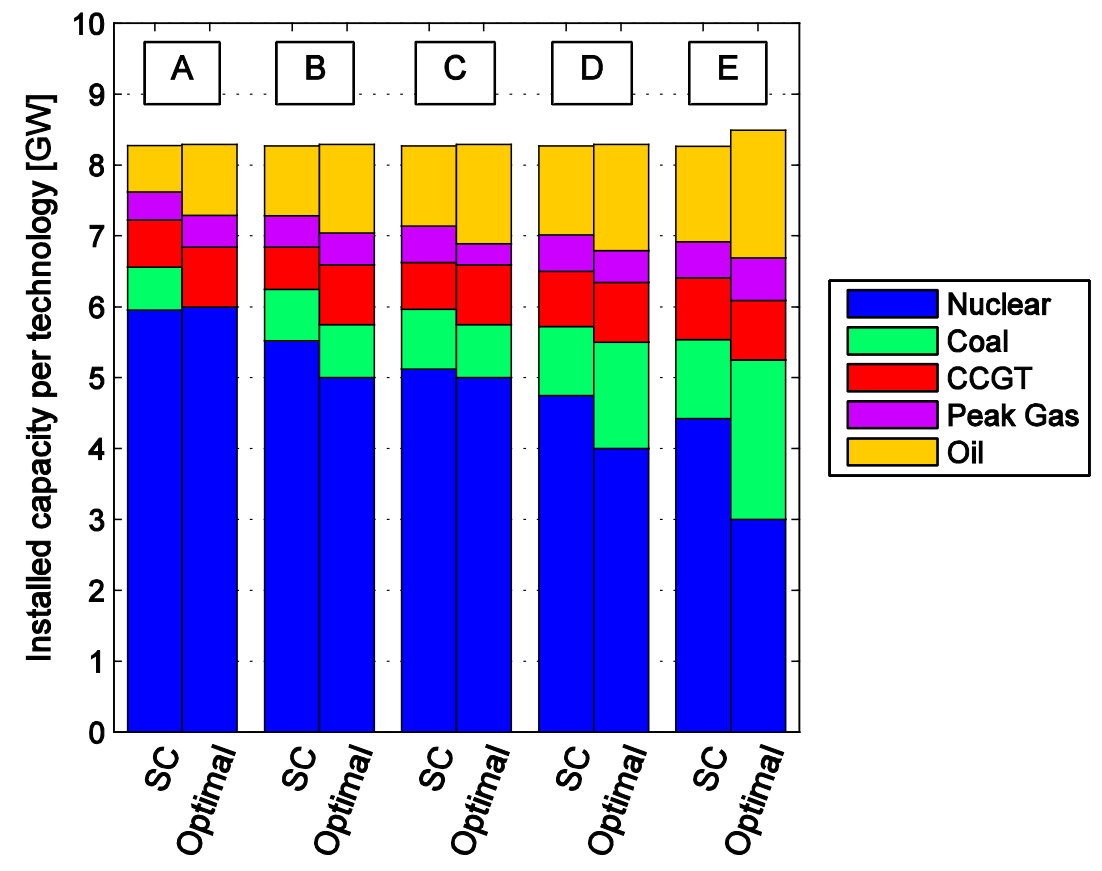

Figure 6. Installed capacity of un-rounded screening curve (SC) model, and optimal set, for each scenario.

For most scenarios, the amount of base load (nuclear) capacity replaced in the optimal set is approximately double the amount of replaced capacity in the screening curve set. This leads to the conclusion that both the economic and operational effect are important when determining the optimal set of generation units as a function of the installed wind capacity: (1) the deployment of RES reduces the residual load and hence creates a shift from base to mid and peak load; (2) this RES 
deployment also increases the volatility of the residual load, so this shift from base to peak load becomes even stronger.

\section{Conclusion}

This paper presents a new comprehensive method for optimal electricity generation system planning, specifically accounting for flexibility required at high penetration levels of renewables. Compared to existing planning models and methods available in the literature, the model developed in this paper combines elements from planning models and technical detailed operational models. The model works on power plant level, and is able to include power plant technical restrictions. This way, power plant flexibility required to deal with RES intermittency can be adequately accounted for. The model can be solved with computation times $<1 \mathrm{~h}$.

A first conclusion relates to the use of the screening curve model and the MILP system planning model as such. As they both provide solutions that are feasible also in operational terms, for cases with or without RES, in theory they can both be used for system planning. The screening curve methodology provides a good estimation of the optimal set and does so in the least amount of computation time. In addition, the perturbation generally converges to the optimal set. Only in the case of the highest wind scenario considered, it occurs that the UC scheduling model employed by the perturbation algorithm could not determine a feasible UC schedule within reasonable calculation time. The MILP system planning model provides in general a worse initial estimation. This model also takes by far the most time to determine an estimated set. The composition of each estimated set changes heavily for each scenario. This is because the model bases its investment decision on a small part of the demand profile. A perturbation of the estimated set converges in general to a nonoptimal set.

As is clear from the perturbation algorithm, taking into account operational constraints through an actual operational UC model affects the optimal solution. Hence, the basic system planning models can be used as such (providing feasible solutions), but need further adaptation to improve their performance (in achieving better solutions). Using the screening curve method to determine the initial set, in combination with the perturbation algorithm and operational UC model finally make up the preferred methodology (i.e., providing the best solutions in computation times remaining below $1 \mathrm{~h})$.

The second conclusion relates to the evolution of the optimal set in function of the amount of installed wind capacity. It is observed that the total amount of installed capacity (excluding wind) is approximately equal for all scenarios. When more wind capacity is installed, a part of the base-load generation units is replaced by mid-load and peak-load generation units. This is explained by two effects. The first effect is the economic effect. An increase of installed wind capacity causes the total residual demand to decrease. A decrease in total residual demand leads to a decrease of the average generation output per generation unit. The decrease of generation output is unfavorable for baseload generation units as they are only economically preferable when their generation output is high. The second effect is the operational effect. An increasing amount of installed wind capacity causes an increase in the volatility of the residual demand. This leads to an increasing need for flexible generation units. This is again unfavorable for the base-load generation units, since their operation flexibility is limited compared to the flexibility of a peak-load generation unit. An examination of the 
simulation results lead to the conclusion that both effects have a significant impact on the composition of the optimal generator set.

\section{Acknowledgements}

E. Delarue is a research fellow of the Research Foundation - Flanders (FWO).

\section{References}

[1] Ortega-Vazquez MA, Kirschen DS. Assessing the impact of wind power generation on operating costs. IEEE Transactions on Smart Grid. 2010; 1:295-301.

[2] Restrepo JF, Galiana FD. Assessing the yearly impact of wind power through a new hybrid deterministic/stochastic unit commitment. IEEE Transactions on Power Systems. 2011; 26:40110.

[3] Ummels BC, Gibescu M, Pelgrum E, Kling WL, Brand AJ. Impacts of wind power on thermal generation unit commitment and dispatch. IEEE Transactions on Energy Conversion. 2007; 22:4451.

[4] Kagiannas AG, Askounis DT, Psarras J. Power generation planning: a survey from monopoly to competition. International journal of electrical power \& energy systems. 2004; 26:413-21.

[5] Alizadeh B, Jadid S. A dynamic model for coordination of generation and transmission expansion planning in power systems. International Journal of Electrical Power \& Energy Systems. 2015; 65:408-18.

[6] Franco JF, Rider MJ, Romero R. A mixed-integer quadratically-constrained programming model for the distribution system expansion planning. International Journal of Electrical Power \& Energy Systems. 2014; 62:265-72.

[7] Stoft S. Power System Economics. New York: Wiley; 2002.

[8] Santos HL, Legey LF. A model for long-term electricity expansion planning with endogenous environmental costs. International Journal of Electrical Power \& Energy Systems. 2013; 51:98105.

[9] Delgado F, Ortiz A, Renedo C, Pérez S, Mañana M, Zobaa AF. The influence of nuclear generation on $\mathrm{CO} 2$ emissions and on the cost of the Spanish system in long-term generation planning. International Journal of Electrical Power \& Energy Systems. 2011; 33:673-83.

[10] Unsihuay-Vila C, Marangon-Lima J, Zambroni de Souza A, Perez-Arriaga I. Multistage expansion planning of generation and interconnections with sustainable energy development criteria: A multiobjective model. International Journal of Electrical Power \& Energy Systems. 2011; 33:258-70.

[11] DeCesaro J, Porter K, Milligan M. Wind Energy and Power System Operations: A Review of Wind Integration Studies to Date. The Electricity Journal. 2009; 22:34-43.

[12] Holttinen H. Wind integration: experience, issues, and challenges. Wiley Interdisciplinary Reviews: Energy and Environment. 2012; 1:243-55. 
[13] Lannoye E, Flynn D, and M. O'Malley. The role of power system flexibility in generation planning. Proceedings of 2011 IEEE Power and Energy Society General Meeting, Detroit, MI, July 2011.

[14] De Jonghe C, Delarue E, Belmans R, D'haeseleer W. Determining optimal electricity technology mix with high level of wind power penetration. Applied Energy. 2011; 88:2231-8.

[15] Nweke Cl, Leanez F, Drayton GR, Kolhe M. Benefits of chronological optimization in capacity planning for electricity markets. Power System Technology (POWERCON), 2012 IEEE International Conference on: IEEE; 2012. p. 1-6.

[16] Delarue E, De Jonghe C, Belmans R, D'haeseleer W. Applying portfolio theory to the electricity sector: Energy versus power. Energy Economics. 2011; 33:12-23.

[17] Short W, Sullivan P, Mai T, Mowers M, Uriarte C, Blair N, Heimiller D, Martinez A. Regional Energy Deployment System (ReEDS). Technical Report NREL/TP-6A20-46534, 2011.

[18] Loulou R, Remne U, Kanudia A, Lehtila A, Goldstein G. Documentation for the TIMES Model: Part I. Energy Technology Systems Analysis Programme (ETSAP), 2005.

[19] Loulou R, Lehtila A, Kanudia A, Remne U, Goldstein G. Documentation for the TIMES Model: Part II. Energy Technology Systems Analysis Programme (ETSAP), 2005.

[20] Kannan R, Turton H. A long-term electricity dispatch model with the TIMES framework. Environmental Modeling \& Assessment. 2013; 18:325-43.

[21] Haydt G, Leal V, Pina A, Silva C. The relevance of the energy resource dynamics in the mid/long-term energy planning models. Renewable Energy. 2011; 36:3068-74.

[22] Pina A, Silva C, Ferrão P. Modeling hourly electricity dynamics for policy making in long-term scenarios. Energy Policy. 2011; 39:4692-702.

[23] Pina A, Silva C, Ferrão P. High-resolution modeling framework for planning electricity systems with high penetration of renewables. Applied Energy. 2013; 112:215-23.

[24] Deane JP, Chiodi A, Gargiulo M, Ó Gallachóir BP. Soft-linking of a power systems model to an energy systems model. Energy. 2012; 42: 303-312.

[25] Ma J, Kirschen DS, Belhomme R, Silva V. Optimizing the flexibility of a portfolio of generating plants. Proceedings of the 17th Power System Computation Conference (PSCC) 2011. p. 22-6.

[26] Palmintier B. Flexibility in Generation Planning: Identifying Key Operating Constraints. Proceedings of the 18th Power System Computation Conference (PSCC) 2014. p. 1-7.

[27] Palmintier B. Incorporating operational flexibility into electric generation planning - Impacts and Methods for System Design and Policy Analysis. PhD thesis, Massachusetts Institute of Technology, February 2013.

[28] Carrión M, Arroyo JM. A computationally efficient mixed-integer linear formulation for the thermal unit commitment problem. IEEE Transactions on Power Systems. 2006; 21:1371-8.

[29] Wood AJ, Wollenberg BF. Power generation, operation, and control: John Wiley \& Sons; 2012.

[30] Nemhauser GL, Wolsey LA. Integer and combinatorial optimization: Wiley New York; 1988.

[31] Delarue E, Cattrysse D, D'haeseleer W. Enhanced priority list unit commitment method for power systems with a high share of renewables. Electric Power Systems Research. 2013; 105:115-23. 
[32] Elia. Grid data, demand. 2013. http://www.elia.be/nl/grid-data/data-download [accessed July 2013].

[33] Elia. Grid data, injected power from wind turbines. 2013. http://www.elia.be/nl/griddata/productie/windproductie [accessed July 2013].

[34] IEA/NEA. Projected Costs of Generating Electricity 2010 edition, International Energy Agency / Nuclear Energy Agency. Paris: OECD Publishing; 2010.

[35] Kazarlis SA, Bakirtzis A, Petridis V. A genetic algorithm solution to the unit commitment problem. IEEE Transactions on Power Systems. 1996; 11:83-92.

[36] Grigg $C$, Wong $\mathrm{P}$, Albrecht $\mathrm{P}$, Allan $\mathrm{R}$, Bhavaraju $\mathrm{M}$, Billinton $\mathrm{R}$, et al. The IEEE reliability test system-1996. A report prepared by the reliability test system task force of the application of probability methods subcommittee. IEEE Transactions on Power Systems. 1999;14:1010-20. 